\title{
Critical assessment of diagnostic value of endomyocardial biopsy Assessment of cardiac biopsy
}

\author{
E. H. MACKAY, ${ }^{1}$ W. A. LITTLER, ${ }^{2}$ AND P. SLEIGHT \\ From the Departments of Pathology and Cardiovascvlar Medicine, Radcliffe Infirmary, Oxford
}

Right or left ventricular endomyocardial biopsy with the Konno or the Olympus bioptome was attempted in 73 patients aged 5 months to 61 years, with 82 per cent success. Light and electron microscopy showed nonspecific features in over half the biopsies with new or diagnostically useful information in only 10 per cent of cases, usually as a result of electron microscopy. The method is safe but is of strictly limited diagnostic value and is likely to be of most help as a research tool in the biochemical study of cardiomyopathies.

In recent years considerable progress in the understanding of disease processes has followed the development of safe and simple biopsy procedures. Nearly all the tissues of the body can be biopsied and the benefits in terms of diagnostic and hence therapeutic precision are clear. In many cases relatively straightforward microscopical examination of biopsy material and correlation with the clinical picture has clarified the classification and pathogenesis of previously obscure conditions. It was confidently expected that the same kind of approach would be successful in dealing with cardiac biopsies from patients with primary heart muscle disease (cardiomyopathy), of unknown cause or association. This is probably a heterogeneous group of diseases of multifactorial aetiology; the diagnosis is usually presumptive in life and made largely by exclusion of other conditions at necropsy. Since the heart has an essentially simple structure with a limited range of responses to a wide variety of insults, the end stage lesions seen at necropsy may give little or no clue to the structural changes in the early stages of the disease or to its aetiology. For these reasons myocardial biopsy early in the course of a cardiomyopathy offers the best hope of making an aetiological diagnosis but at the same time this early stage in the disease process may be the most difficult to interpret morphologically.

None of the early methods of cardiac biopsy has

${ }^{1} \mathrm{E}$. H. MacKay is in receipt of a locally organised research grant. 'Present address: East Birmingham Hospital, Bordesley Green East, Birmingham.

Received for publication 24 January 1977 gained widespread or lasting acceptance. Limited thoracotomy and cardiac drill biopsy as reported by Weinberg et al. (1958) and later by Sutton et al. (1964) can never be considered a routine investigation even though larger quantities of tissue are obtained and inspection of the heart and coronary vessels is possible. Various forms of percutaneous needle biopsy have been reported by Sutton and Sutton (1960), Bercu et al. (1964), and Shirey et al. (1972) but all have been associated with various unpleasant or even dangerous complications ranging from temporary arrhythmias to cardiac tamponade, occurring in 8 out of 198 patients in the last series. Intracardiac needle biopsy of the ventricular septum as reported by Bulloch et al. (1965) was relatively free of complications and supplied quite satisfactory material for ultrastructural studies.

The present phase of the cardiac biopsy programme began in 1962 with the development of the endomyocardial bioptome by Sakakibara and Konno in Japan. Since that time the Japanese workers have biopsied well over 500 patients and obtained samples safely from all chambers of the heart. Only a few other relatively small series of endomyocardial biopsies have been reported, the largest being those of Somers et al. (1971) with 64 cases and Olsen (1974) with 67 cases. The majority of these biopsies were taken from the right ventricle, with the main emphasis on light microscopy, minimal information being recorded from other forms of study. However, recently a more flexible, though smaller, biopsy instrument, the King's bioptome, has been developed (Richardson, 1974) 
from a modified Olympus bronchial biopsy forceps. Experience with this instrument used to compare right and left ventricular biopsies has been reported by Brooksby et al. (1974), with 100 per cent success in obtaining biopsy specimens from the left ventricle and 90 per cent success from the right ventricle. However, 2 patients in this series undergoing right ventricular biopsy with the newer catheter developed a small haemopericardium, though this did not require active intervention.

The present paper is a critical account of our experience of endomyocardial biopsy at the Radcliffe Infirmary, Oxford, and our conclusions regarding the clinical diagnostic usefulness of light and electron microscopical examination of these biopsies.

\section{Material and methods}

Endomyocardial biopsy was attempted in 73 patients with clinical features suggesting the possibility of cardiac muscle disease. All had undergone full cardiac catheterisation studies including left ventricular and coronary artery angiography in most cases. In 62 cases biopsy was attempted from the apex of the right ventricle or right side of the interventricular septum with the Konno or Olympus endomyocardial bioptome, which was introduced via the saphenous vein in the inguinal region and guided into the right ventricle under fluoroscopic control. In 11 patients left ventricular biopsies were obtained with the Olympus instrument, through a large atrial septal defect in one patient and by retrograde arterial catheterisation through a long sheath in the others. A total of 79 usable samples of cardiac muscle was obtained from 60 patients for light and electron microscopy, plus a further 19 samples for virology and histochemistry.

In the early stages of this study the samples were placed immediately into 10 per cent neutral formol saline and processed into paraffin wax for histology. Sections were stained routinely with haematoxylin and eosin, periodic acid Schiff, with or without diastase digestion, Verhoeff/van Gieson for elastic tissue and collagen, Lendrum's Martius scarlet blue for fibrin, phosphotungstic acid haematoxylin, and other stains as required. The histological and electron microscopical sections were not examined 'blind' as the pathologist is part of the cardiac team and was usually aware of the initial clinical diagnosis from joint discussion at case conferences. Fibre hypertrophy was assessed by using an eyepiece micrometer to measure the minimum diameter of an average of 80 crosssectioned fibres in each biopsy. In the majority of cases part of the specimen or a separate sample was fixed in ice cold 4 per cent glutaraldehyde, post fixed in 1 per cent osmium tetroxide, and processed into araldite for electron microscopy. Ultrathin sections of selected areas were cut on an LKB Mk. III Ultratome, stained with uranyl acetate and lead citrate, and examined in a Philips 301 electron microscope. In the latter half of the series the samples for microscopy were processed as above after a period of prefixation for 5 minutes in ice cold 1 per cent paraformaldehyde in $0.2 \mathrm{M}$ sucrose in phosphate buffer $\mathrm{pH} 7 \cdot 4(\mathrm{PF} / \mathrm{S})$. It had been found that the addition of this step almost completely prevented the formation of artefactual hypercontraction bands in the muscle fibres (see Discussion).

In a small number of cases part of the sample was taken for histochemistry. The muscle was surrounded by OCT embedding compound ${ }^{1}$ and quenched in isopentane precooled to $-160^{\circ} \mathrm{C}$ in liquid nitrogen. Cryostat sections cut at $-20^{\circ} \mathrm{C}$ were incubated for demonstration of succinic and lactic dehydrogenase, phosphorylase, monoamine oxidase, and myosin ATPase, using standard methods and reagents (Chayen et al., 1973).

Attempts were made to isolate viruses from 13 cases using part of the sample ground up in culture medium and inoculated into monkey kidney and HeLa cell cultures and newborn mice. Blood was taken from all patients with a provisional diagnosis of congestive cardiomyopathy or myocarditis, and screened for Coxsackie B, cytomegalovirus, Q fever, toxoplasma, and influenza antibodies.

\section{Results}

Of the 73 patients in this series, 40 were male and 33 female; their ages ranged from 5 months to 61 years. Usable samples of cardiac muscle were obtained from 60 of these patients but in the remaining 13 patients no myocardial tissue could be obtained after 2 or 3 attempts giving an overall biopsy success rate of 82 per cent.

Table 1 summarises the initial clinical diagnoses and biopsy findings in the 60 successfully biopsied patients, with an indication of the value of the biopsy in each clinical category. Also shown are the numbers of patients in each category from whom a biopsy could not be obtained. Cardiomyopathy and myocarditis in various forms were the principal initial clinical diagnoses in nearly two-thirds $(63 \%)$ of the successfully biopsied cases. Ischaemic heart disease in adults and possible endocardial fibroelastosis associated with congenital heart disease in children were the main alternatives. The distribution of the final clinical diagnoses is ${ }^{1}$ Ames Company, Division Miles Laboratory Inc. 
Table 1 Summary of clinical and pathological diagnoses, value of biopsy, and necropsy findings

\begin{tabular}{|c|c|c|c|c|c|c|}
\hline \multirow{2}{*}{$\begin{array}{l}\text { Initial clinical diagnoses } \\
\begin{array}{l}\text { Hypertrophic cardiomyopathy } \\
\text { (incl. HOCM) }\end{array}\end{array}$} & \multirow{2}{*}{$\frac{\text { Cases }}{13(2)}$} & \multicolumn{2}{|l|}{ Pathological diagnoses } & \multicolumn{2}{|c|}{ Contribution of biopsy } & \multirow{2}{*}{$\frac{\text { Notes }}{1 \text { died (no necropsy) }}$} \\
\hline & & $\begin{array}{l}\text { HOCM } \\
\text { Possible HOCM } \\
\text { Hypertrophy } \\
\text { Non-specific } \\
\text { Normal } \\
\text { Viral myocarditis }\end{array}$ & $\begin{array}{l}3 \\
2 \\
4 \\
2 \\
1 \\
1\end{array}$ & $\begin{array}{l}\text { Diagnostic } \\
\text { Helpful } \\
\text { Limited help } \\
\text { Confirmatory } \\
\text { No help }\end{array}$ & $\begin{array}{l}1 \\
4 \\
1 \\
2 \\
5\end{array}$ & \\
\hline $\begin{array}{l}\text { Cardiomyopathy (incl. CoCM, } \\
\text { restrictive CMP, familial } \\
\text { CMP) }\end{array}$ & $10(1)$ & $\begin{array}{l}\text { CoCM } \\
\text { Hypertrophy } \\
\text { Non-specific } \\
\text { Normal }\end{array}$ & $\begin{array}{l}1 \\
3 \\
4 \\
2\end{array}$ & $\begin{array}{l}\text { Limited help } \\
\text { Confirmatory } \\
\text { No help } \\
\text { Misleading }\end{array}$ & $\begin{array}{l}2 \\
1 \\
6 \\
1\end{array}$ & $\begin{array}{l}3 \text { died. Necropsies showed LV } \\
\text { fibrosis (case 5) and CoCM + } \\
\text { ASD (1 case) (no necropsy in 1) }\end{array}$ \\
\hline Puerperal CMP & 4 & $\begin{array}{l}\text { CoCM } \\
\text { Hypertrophy } \\
\text { Normal }\end{array}$ & $\begin{array}{l}1 \\
2 \\
1\end{array}$ & $\begin{array}{l}\text { Confirmatory } \\
\text { No help }\end{array}$ & $\begin{array}{l}1 \\
1 \\
3\end{array}$ & \\
\hline Alcoholic CMP & 3 & $\begin{array}{l}\text { Alcoholic CMP } \\
\text { ? Myocarditis } \\
\text { ? Myopathy }\end{array}$ & $\begin{array}{l}1 \\
1 \\
1\end{array}$ & $\begin{array}{l}\text { Helpful } \\
\text { Limited help } \\
\text { Confirmatory }\end{array}$ & $\begin{array}{l}1 \\
1 \\
1\end{array}$ & $\begin{array}{l}2 \text { died. Necropsy showed CoCM } \\
(1 \text { case) (no necropsy in } 1 \text { ) }\end{array}$ \\
\hline ? Myocarditis & $6(1)$ & $\begin{array}{l}\text { Non-specific } \\
\text { Hypertrophy } \\
\text { Normal }\end{array}$ & $\begin{array}{l}4 \\
1 \\
1\end{array}$ & $\begin{array}{l}\text { Helpful } \\
\text { Limited help } \\
\text { No help }\end{array}$ & $\begin{array}{l}2 \\
1 \\
3\end{array}$ & $\begin{array}{l}2 \text { died. Necropsies showed CoCM } \\
(1 \text { case) and RHD only (case } \\
44)\end{array}$ \\
\hline $\begin{array}{l}\text { Heart block (biopsy to exclude } \\
\text { sarcoidosis) }\end{array}$ & $3(1)$ & $\begin{array}{l}\text { ? IHD } \\
\text { Hypertrophy } \\
\text { Normal }\end{array}$ & $\begin{array}{l}1 \\
1 \\
1\end{array}$ & Limited help & 3 & $\begin{array}{l}\text { (none shown to } \\
\text { have sarcoid) }\end{array}$ \\
\hline Ischaemic heart disease & $4(1)$ & $\begin{array}{l}\text { ? IHD } \\
\text { Hypertrophy }\end{array}$ & $\begin{array}{l}3 \\
1\end{array}$ & $\begin{array}{l}\text { Limited help } \\
\text { Confirmatory }\end{array}$ & $\begin{array}{l}1 \\
3\end{array}$ & 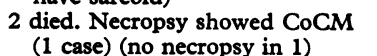 \\
\hline Skeletal myopathy & 2 & $\begin{array}{l}\text { Mitochondrial myopathy } \\
\text { ? Myopathy }\end{array}$ & 1 & $\begin{array}{l}\text { Diagnostic } \\
\text { Confirmatory }\end{array}$ & $\begin{array}{l}1 \\
1\end{array}$ & 1 died (no necropsy) \\
\hline $\begin{array}{l}\text { Congenital heart disease (biopsy } \\
\text { to exclude endocardial } \\
\text { fibroelastosis) }\end{array}$ & $8(5)$ & $\begin{array}{l}\text { Endocardial fibroelastosis } \\
\text { ? Myopathy } \\
\text { Hypertrophy } \\
\text { Normal }\end{array}$ & $\begin{array}{l}1 \\
1 \\
2 \\
4\end{array}$ & $\begin{array}{l}\text { Diagnostic } \\
\text { Limited help } \\
\text { No help }\end{array}$ & $\begin{array}{l}1 \\
6 \\
1\end{array}$ & 1 died: necropsy TGV \\
\hline $\begin{array}{l}\text { Rheumatic heart disease and } \\
\text { others }\end{array}$ & $7(2)$ & $\begin{array}{l}\text { Hypertrophy } \\
\text { Non-specific } \\
\text { Normal }\end{array}$ & $\begin{array}{l}5 \\
1 \\
1\end{array}$ & $\begin{array}{l}\text { Helpful } \\
\text { Limited help }\end{array}$ & $\begin{array}{l}1 \\
3\end{array}$ & $\begin{array}{l}1 \text { died: necropsy postoperative } \\
\text { endocarditis }\end{array}$ \\
\hline & & & & No help & 3 & \\
\hline
\end{tabular}

Cases

$60(13)$

Figures in parentheses indicate number of patients in each clinical group in whom a biopsy could not be obtained.

CMP, cardiomyopathy; HOCM, hypertrophic cardiomyopathy \pm obstruction; CoCM, congestive cardiomyopathy; IHD, ischaemic heart disease; ASD, atrial septal defect; TGV, transposition of great vessels.

*Previously reported by MacKay et al. (1976).

seen in Fig. 1 which shows that cardiomyopathy and myocarditis were the main final diagnoses in only 53 per cent of the successfully biopsied patients after completion of all investigations including angiocardiography and biopsy. The contribution of the biopsy findings to the final diagnosis obviously varies considerably and has to be balanced against clinical, catheter, and angiographic evidence in each case. Taking the successful group as a whole, the biopsy was thought to be diagnostic or positively helpful in 15 per cent of these cases (Table 1) or 12 per cent of the full series.

The pathological diagnoses in this series are summarised in Table 2 and it will be clearly seen from this and from Fig. 1 that the range and specificity of morphological diagnosis is distinctly limited. As mentioned above, this is partly because of the limited response available to the myocardium in various disease states. Thus in a third of right ventricular biopsies no more precise classification than fibre hypertrophy could be offered. In the absence of other changes this was of very little

Table 2 Final morphological diagnosis after successful biopsy

\begin{tabular}{lcc}
\hline & No. of patients & Per cent \\
\hline No abnormality & 10 & 16 \\
Myocarditis & 2 & 3 \\
Ischaemic heart disease & 4 & 7 \\
HOCM & 5 & 8 \\
CoCM & 2 & 3 \\
Other diagnoses & 6 & 11 \\
Non-specifict & 31 & 52 \\
\hline & 60 & 100
\end{tabular}

*Includes endocardial fibroelastosis, mitochondrial and skeletal myopathies, and alcoholic cardiomyopathy.

tIncludes various degrees of hypertrophy and minor endocardial or interstitial fibrosis. 
value and correlated poorly with clinical and electrocardiographic evidence of right ventricular hypertrophy or with the right ventricular pressures on catheterisation. As with so many other biopsy procedures a normal result, seen in 16 per cent of this series, must be treated with caution as it does not necessarily exclude disease elsewhere in the heart.

A good correlation between light and electron microscopy appearances was seen in two-thirds $(63 \%)$ of the biopsies and a fair degree of agreement in a further quarter $(26 \%)$. More important however was the positive diagnostic information obtained by electron microscopy on three of the remaining cases in which light microscopy did not reveal any specific features. On the other hand, the relation between final clinical diagnoses and the final pathological diagnoses as seen in Fig. 1 shows quite clearly the non-specificity of the latter in just over half of these cases.

The complete ultrastructural analysis of this series will be reported later but some of the main electron microscopical features may be considered here. In general, fibre hypertrophy was more easily assessed by light microscopy as the ultrastructural features are non-specific and include a generalised increase in number of mitochondria and prominent nucleoli. The so-called 'cardiac villi' are not evidence of myofibre hypertrophy but merely reflect wrinkling of the sarcolemma caused by intense fibre contraction. Myofibrillar disarray was seen in hypertrophic cardiomyopathy (HOCM), congestive cardiomyopathy (CoCM), dystrophic hearts, familial heart block, and a case of myocarditis; when seen alone it is non-specific. When seen to a major degree with light microscopical evidence of myofibre disorder it is virtually pathognomonic of HOCM and these biopsies can be diagnostically useful. Conversely, biopsies from patients with clinical and catheterisation evidence of CoCM rarely show specific changes and alterations in size and number of mitochondria with thin disappearing fibrils are suggestive but not truly diagnostic. No consistent morphological changes have been seen which might provide clues to the aetiology in individual cases of CoCM but the numbers are small and the aetiology is very likely to be multifactorial. Though disappointing so far, it is hoped that biopsy in this group in particular will, in the longer term, provide structural and clinical evidence of the mode of origin of this condition.

Histochemical tests were disappointing largely as a result of the small size of the specimen leading to technical problems and uncertainties in interpretation of results. No consistent changes were found in any enzyme system but the numbers so far are too small to permit firm conclusions.

Although many of the patients with CoCM had an acute onset with an influenza-like illness, none

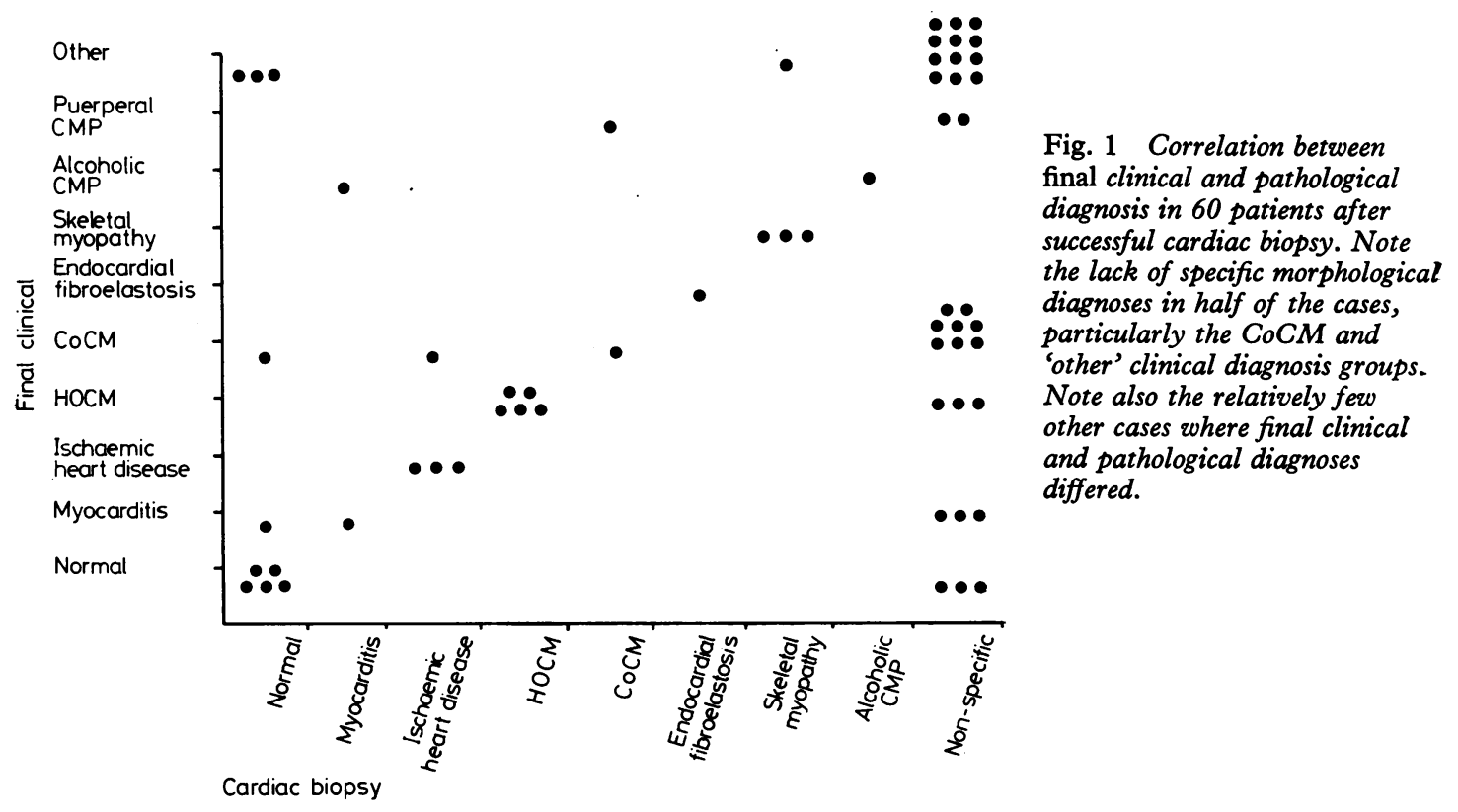

Fig. 1 Correlation between final clinical and pathological diagnosis in 60 patients after diagnoses in half of the cases, particularly the CoCM and 'other' clinical diagnosis groups. and pathological diagnoses differed. 
had abnormal serological results suggesting recent viral infection. Unfortunately, in the one patient with ultrastructural changes suggesting viral myocarditis, no acute phase serum sample was obtained but at follow-up there is still evidence of left ventricular enlargement and a gallop rhythm has developed. Similarly all the samples submitted for virus culture gave completely negative results.

In the course of the study 13 patients died, from one month to one year after biopsy, and 8 came to necropsy. Positive biopsies had been obtained in 9 of the patients who died, but of the 8 coming to necropsy, 3 were failed biopsies, and in 4 necropsy confirmed the clinical and biopsy findings. The biopsy site was positively identified in 2 patients (cases 5 and 44) and, as shown in Fig. 2 and 3, was healing normally with no mural thrombus, no excessive local damage, and no likelihood of perforation or embolus formation.

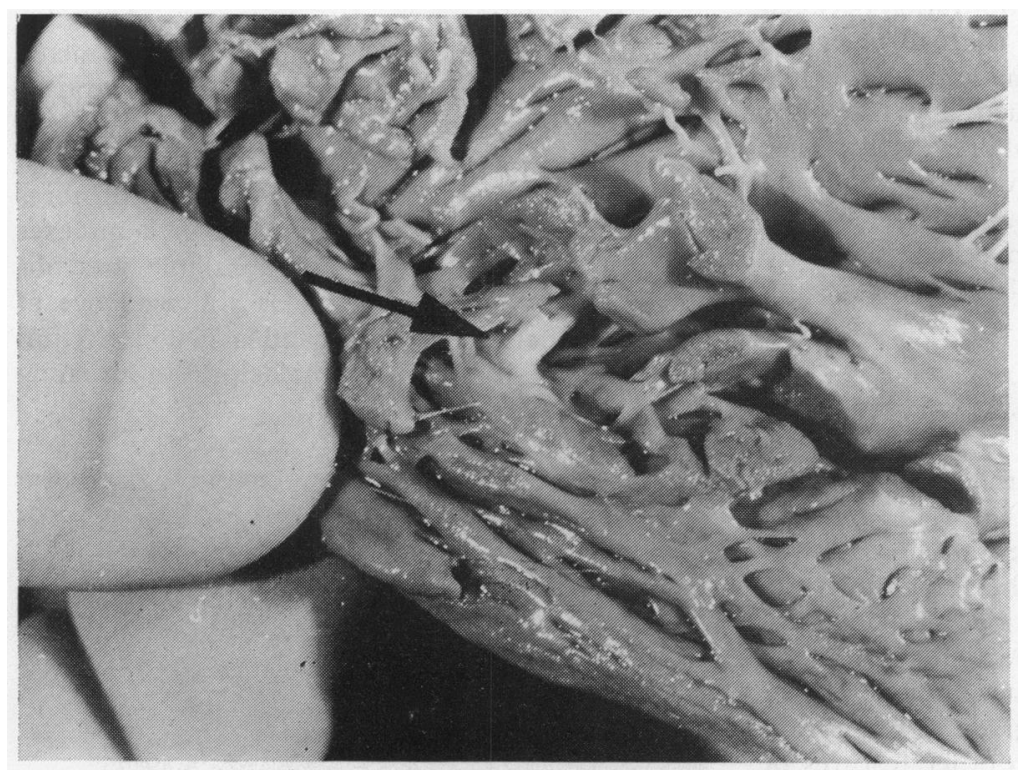

Fig. 2 Healed $R V$ biopsy site (arrowed) from case 5, 33 days after biopsy with Konno bioptome.

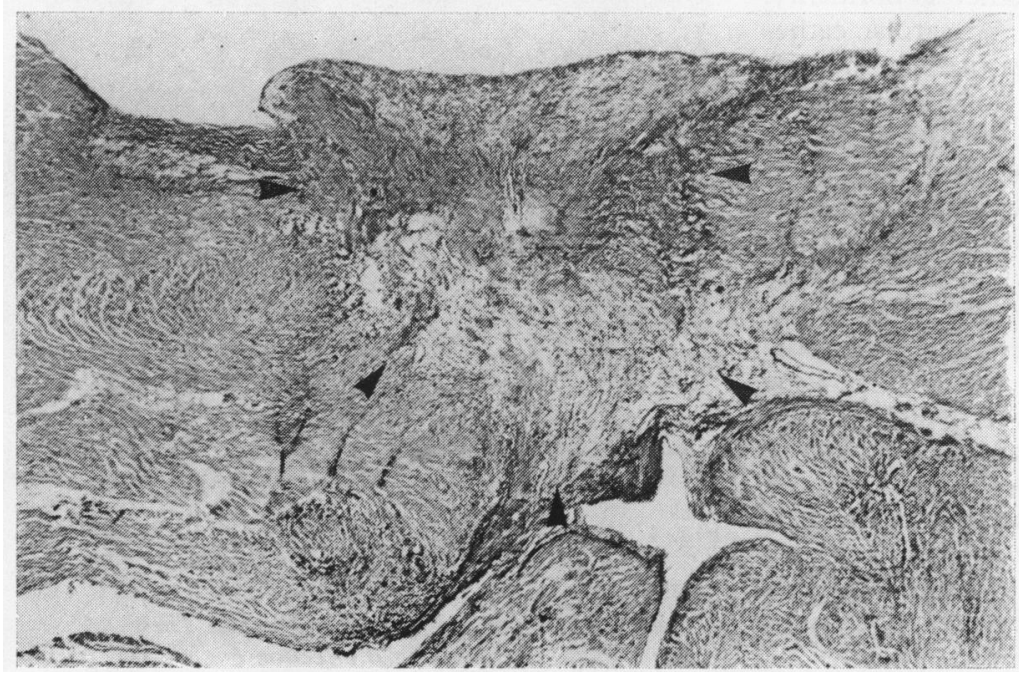

Fig. 3 Histological section of $R V$ biopsy site (case 5) showing healing scar tissue (arrowed) and lack of surface thrombus. (Verhoeff/Van Gieson. × 36.) 


\section{Illustrative case reports}

\section{CASE 1: UNEXPECTED MYOCARDITIS}

This 52-year-old woman had developed unexplained gross congestive cardiac failure which responded to routine diuretic treatment. There were no valve lesions. She had a moderate alcohol intake and this was considered a possible cause of an otherwise unexplained cardiomyopathy. Cardiac catheterisation was unhelpful but right ventricular cardiac biopsy showed an unexpected myocarditis with extensive mononuclear cell infiltration between the fibres which were otherwise normal. Over the following three years she has made a steady improvement, has now discontinued all treatment, and is symptom-free; her gallop rhythm has disappeared, and there are no other abnormal physical signs. In retrospect it appears likely that she was suffering from a myocarditis (cause unknown) and the biopsy was helpful in suggesting this diagnosis.

\section{CASE 5: MISLEADINGLY NORMAL RIGHT VENTRICULAR BIOPSY}

A 16-year-old girl presented with one episode of retrosternal pain associated with an upper respiratory tract infection. Her aspartate transaminase was raised and she was referred to the Radcliffe Infirmary with a diagnosis of a possible viral myocarditis. Her sister had died unexpectedly at the age of 17 one year earlier. Our patient had no abnormal physical signs apart from a grade $2 / 6$ ejection systolic murmur. There were no other features of hypertrophic cardiomyopathy or aortic valve disease. Electrocardiogram showed a right bundlebranch block pattern, but no other abnormality. The chest $x$-ray film was normal. At cardiac catheterisation the end-diastolic pressure in the left ventricle was raised $(13 \mathrm{mmHg})$ but there were no abnormalities on angiography. In particular the left ventricular cavity was small and contracted well. The coronary vessels were normal. Cardiac biopsy (right ventricle) was completely normal.

The patient died suddenly while at home one month after the investigation, and at necropsy there was diffuse widespread scarring in the middle third of the left ventricular wall (we later saw the postmortem histology from her sister which showed the same finding). The right ventricular muscle at necropsy was normal and the biopsy site was well healed (Fig. 2, 3). This case illustrates the pitfalls of a small and localised biopsy when no abnormality is shown on microscopy. Incidentally it is highly unlikely that a left ventricular biopsy would have shown any abnormality either.
CASE 23: UNEXPECTED ?VIRAL MYOCARDITIS

This 48-year-old man had no symptoms. At an insurance medical examination chest $x$-ray showed left ventricular enlargement and an electrocardiogram showed attacks of paroxysmal tachycardia and frequent ventricular ectopic beats. Examination was otherwise negative. Cardiac catheterisation was complicated by a period of ventricular fibrillation before the biopsy. Pressures in the left side of the heart were in the normal range and there was no obvious obstruction in the outflow tract of the left ventricle. A left ventricular angiogram showed a moderately enlarged left ventricle which contracted well. Coronary angiogram showed normal coronary vessels. A right ventricular cardiac biopsy showed electron microscopical appearances typical of recent viral infection with disorganisation of fibres and formation of membrane-vesicle complexes (Fig. 4). No acute phase serum sample was obtained, but at follow-up there is still evidence of left ventricular enlargement and a gallop rhythm has developed. This biopsy was helpful in revealing

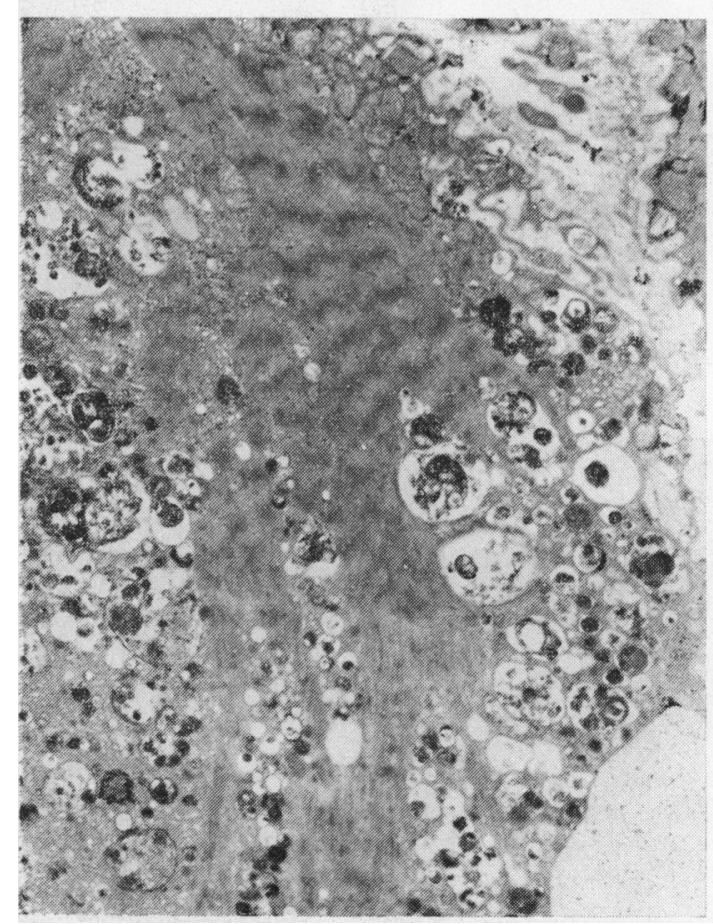

Fig. 4 Case 23. Man 48 years. Electron micrograph of $R V$ biopsy showing disorganisation of myofibrils, blurring of Z-bands, loss of mitochondria, and formation of electron-dense membrane-vesicle complexes. These features suggest a recent viral infection $(\times 5300)$. 
a viral myocarditis which had been previously unsuspected.

\section{CASE 26: BIZARRE MITOCHONDRIA}

A 10-year-old boy was known to have an unusual type of proximal skeletal myopathy. A cardiac murmur had been known to be present from the age of 5 years and he had occasional episodes of dizziness. His electrocardiogram was abnormal with a short PR interval $(0.10 \mathrm{~s})$, narrow $Q R S$ complexes, and abnormal $Q$ and $R$ waves in the chest leads. Chest $x$-ray film showed cardiomegaly with a globular contour. Electromyography was abnormal suggesting primary muscle disease. Cardiac catheterisation showed normal right and left sided pressures with no evidence of ventricular outflow gradients at rest or after provocation. The left ventriculogram showed a large left ventricle which contracted normally with no asymmetrical hypertrophy. Right ventricular cardiac biopsy disclosed a unique abnormality of mitochondria which showed increased size and numbers with ring-shaped forms. Skeletal muscle biopsy was entirely normal. The clinical implications of this case are not clear; it may indicate an early mitochondrial structural abnormality which is not seen in later stages of the disease. This case has been reported fully elsewhere (MacKay et al., 1976).

CASE 44: NEGATIVE VIROLOGY HELPFUL

A 50-year-old man with long-standing rheumatic heart disease had an influenza-like illness in 1972 followed by the onset of cardiac failure which improved on treatment. In May 1974 he was admitted again in heart failure and a cytomegalovirus antibody titre was 1:64. This had risen by July 1974 to $1: 1024$ and cytomegalovirus had been isolated from the urine. During the course of cardiac catheterisation in August 1974 a right ventricular biopsy was taken which showed no evidence of myocarditis and no cytomegalovirus was grown from it on culture. Cardiac catheterisation had shown a small mitral gradient and mitral regurgitation. He died in October 1974 in severe cardiac failure. At necropsy the immediate cause of death was pulmonary embolism as a result of his cardiac failure and erratic anticoagulant control. The mitral valve showed the typical features of rheumatic heart disease with stenosis and regurgitation. No histological or virological evidence of cytomegalovirus infection was found in the heart or other organs though a viral myocarditis had been considered as a possible cause of his deterioration before the cardiac biopsy. The biopsy report was thus vindicated by the necropsy findings.
CASE 49: CONFIRMATION OF SUSPECTED ENDOCARDIAL FIBROELASTOSIS

This 9-month-old girl was the youngest of three children, the eldest of whom had died of endocardial fibroelastosis. She presented with recurrent respiratory tract infections leading to congestive cardiac failure, and was shown to have incontinentia pigmenti. Cardiac catheterisation showed a dilated poorly contracting left ventricle and a right ventricular biopsy confirmed the presence of endocardial fibroelastosis, the endocardium being $120 \mu$ thick. She subsequently improved with conservative treatment. Biopsy was helpful in this case by positively establishing the presence of endocardial fibroelastosis and thereby aiding genetic counselling to this family.

\section{CASE 68: HELPFUL NEGATIVE HISTOLOGY}

A 32-year-old army officer had experienced pulmonary oedema after two weeks on Mount Kilimanjaro. After transfer to lower altitude he took some time to recover and had a productive cough. Afterwards he had recurrent chest pain not always related to exertion. He also had minor electrocardiographic abnormalities with ST depression and $T$ wave flattening. Because of the long delay between his arrival at altitude and the pulmonary oedema, a diagnosis of high altitude pulmonary oedema was thought unlikely and the possibility of a viral myocarditis was considered. Cardiac catheterisation including coronary angiography was entirely normal. It was extremely helpful (with the reservations mentioned above) for his promotion prospects that microscopy and virological study of a cardiac biopsy were also completely negative. After this reassurance he returned to completely normal duties and has been well for a subsequent 14 months.

\section{Discussion}

Our experience of endomyocardial biopsy of the heart confirms that of the Japanese and other workers (Sekiguchi and Konno, 1969; Konno et al., 1971; Somers et al., 1971) that the procedure is safe with, in our hands, no morbidity and no mortality. This applies equally to adults and children and to the use of the Konno and Olympus bioptomes. We have had no post-biopsy complications such as pulmonary emboli, arrhythmia, or cardiac perforation, and Fig. 2 and 3 show quite clearly that the biopsy site has healed without mural thrombus formation. Our experience with left heart biopsy is limited at this stage and we are not yet convinced that it offers any real diagnostic advantage over the right ventricular approach (Brooksby et al., 1974). 
We have deliberately tried to restrict the use of the bioptome to one or two experienced investigators as we feel that this increases both the safety and the success rate of this procedure. It is perhaps significant that in the early stages of the study when two operators (W.A.L., P.S.) used the Konno bioptome exclusively, a success rate of 90 per cent was achieved. Later, when the Olympus instrument was used more frequently and other operators began to learn the technique the overall success rate fell to 82 per cent. Failure to obtain a biopsy was due to inability to pass the bioptome into the heart for anatomical reasons in four patients. In one infant, the veins were too small and in one adult they were distorted by bilateral inguinal hernia repairs. It was impossible to pass the stiffer Konno bioptome into the venous ventricle of one adult with a corrected transposition of the great vessels and the attempt was abandoned in another adult with anomalous pulmonary venous drainage and ankylosing spondylitis on account of back discomfort from a long catheter session. Only one attempt was terminated by local discomfort.

The remaining 8 failures were largely the result of inexperience of the operator, but on two occasions mechanical problems causing incomplete closure of the bioptome jaws were partly responsible. We have also deliberately limited the use of this technique to those patients in whom the diagnosis was still obscure after clinical examination and cardiac catheterisation. This undoubtedly accounts for the relatively small number of biopsies in the 3-year period, averaging 2 cases per month, and constituting only 7 per cent of the total patients catheterised in the same period.

It is obvious from Table 1 and Fig. 1 that the light and electron microscopical appearances of the biopsy specimen are of limited help to the clinician. In our opinion, Olsen (1974), who claimed a 65 per cent diagnostic success rate in 67 patients, the majority of whom had undergone right ventricular biopsy, gives an over optimistic impression of the yields of biopsy. Thirty-four of his patients had congestive cardiomyopathy and we do not believe that this condition has sufficiently characteristic histological features to warrant a precise pathological diagnosis. This, therefore, remains in effect a diagnosis by exclusion, an unsatisfactory state of affairs. It will be further evident from Table 1 and Fig. 1 that little additional diagnostic information is gained from patients with clinical and catheter evidence of typical HOCM and we feel that routine biopsy is not indicated in these cases where the diagnosis is obvious from clinical examination and catheterisation. In only 3 of our patients could the biopsy make a diagnosis which could not have been arrived at by any other means.

Normal morphological appearances of the biopsy specimen obviously cannot exclude disease elsewhere in the myocardium and caution should be exercised in such a case. An illustrative example was case 5 mentioned above. This patient and her family had been reassured on the strength of an unremarkable catheterisation study and a normal biopsy, thus illustrating the potentially misleading value of a normal biopsy. On the other hand, negative biopsies may be helpful in some cases, for example in excluding endocardial fibroelastosis when normal endocardium is shown on biopsy. In general, however, and with these possible exceptions, the biopsy appearances alone were of no prognostic significance, and once again were no more than complementary to clinical and catheterisation data.

The size of the individual biopsy specimen was an important limiting factor in deciding which investigation or analyses should be undertaken and whenever a small biopsy only could be obtained it was preferentially committed for araldite embedding. This had the advantage of producing thin sections for light microscopy with good definition and avoidance of shrinkage artefact which in this series averaged 22 per cent when myofibre diameters in araldite and paraffin sections were compared. In general the Konno bioptome was capable of producing a single sample up to $3 \mathrm{~mm}$ in diameter whereas the Olympus samples were rarely more than $1.5 \mathrm{~mm}$ diameter (Fig. 5). Thus a single Olympus biopsy specimen was not often suitable for paraffin embedding in view of the problems with shrinkage, and was quite inadequate for serious histochemical examination. Orientation of such small samples is clearly difficult if not impos-

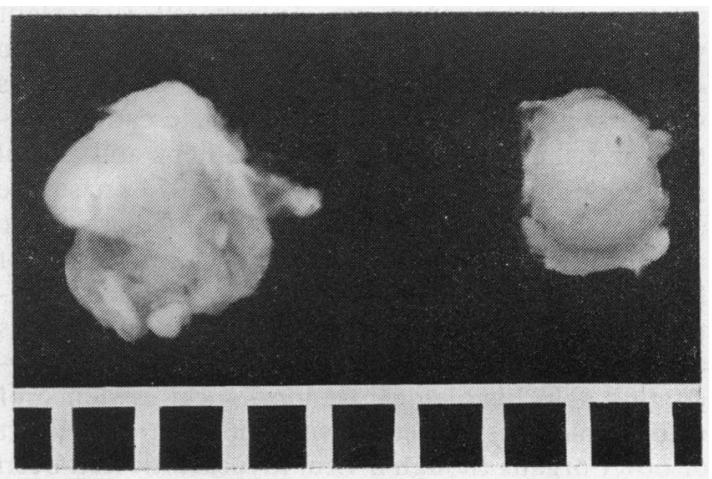

Fig. 5 Comparison of size of biopsies obtained with the Konno (left) and Olympus (right) bioptomes. Scale bars represent $1 \mathrm{~mm}$ intervals. 
sible and further division after fixation before embedding in araldite improved the chances of cutting fibres in longitudinal and transverse planes plus an endocardial surface. Multiple biopsies would produce the same effect but are only obtainable with the long sheath technique and are probably not justified for purely morphological examination. They are, however, essential to obtain an adequate amount of tissue for biochemical assays or cell fractionation. It is possible that multiple biopsies obtained through a sheath tend to be taken from the same site and thus lead to a relatively high perforation rate (Brooksby et al., 1974).

Traumatic damage to the periphery of the biopsy had a proportionately greater effect on the smaller biopsies but was rarely of a degree severe enough to prevent histological assessment. In the early stages of the study artefactual fixation-induced hypercontraction bands in the fibres were a more serious problem. We found empirically that this could be almost completely prevented by the introduction of a 5-minute prefixation period in ice-cold 1 per cent $\mathrm{PF} / \mathrm{S}$. This fixative contains tetrasodium pyrophosphate and has been used by Khan et al. (1972) for optimal preservation of myosin ATPase before its histochemical localisation. It was thought to work by competitive inhibition of ATP breakdown by the myosin ATPase thereby preventing myofibre contraction. This prefixation procedure had no unwanted side effects on the light microscopy, apart from the retention of more glycogen than usual. On electron microscopy prefixation appears to cause some tubular dilatation (mainly sarcoplasmic reticulum) and prominence of pinocytic vesicles but no other abnormality and there is no effect on processing or staining.

A distinction has to be made between the clinical or diagnostic value of cardiac biopsy and its purely research value when used in the search for aetiological factors in congestive cardiomyopathy. So far, as shown by our results and those of other workers, the truly diagnostic yield is low if results are examined critically. Useful information, sufficient to change the diagnosis or to alter significantly the treatment or prognosis, is only obtained in about 10-15 per cent of biopsies even from a highly selected series. On occasions when the biopsy merely confirms a clinically obvious diagnosis, it cannot be considered clinically helpful. In our experience however, the diagnostic success could not have been achieved by other means and thus should not be lightly dismissed.

From a research point of view cardiac biopsy offers the only method of actually examining cardiac muscle in life before end stage pathological changes have developed. Biopsy in the early stages of disease may thus be the only way of showing causes of CoCM by morphological or biochemical examination. Histochemical examination is of very limited value because the techniques available for enzyme or substrate demonstration are relatively crude and methods are not available for the critical rate limiting enzymes in various metabolic cycles. It is of no help at all to demonstrate the presence of myosin ATPase for example if it cannot be quantitated. Similarly some of the minor histochemical variations reported in dehydrogenase enzymes are probably the result of technical factors rather than genuine pre-biopsy alterations in cellular enzyme content or activity. The use of quantitative methods for the dehydrogenases by formazan production from tetrazolium salts and measurement of the amounts produced with a spectrophotometer (Altmann, 1969) is one approach which may be able to settle this problem. An expression of the results in terms of oxygen usage may permit easier comparison with other methods of the assessment of cardiac function.

Biochemical examination of biopsy material is now possible but is also limited by the small quantities of muscle obtained, usually 1 to $3 \mathrm{mg}$ per biopsy. Peters et al. (1976) showed a decrease in myosin ATPase and an increase in lactate dehydrogenase in biopsies from patients with poor left ventricular function caused by valvular heart disease, but how, why, and when these changes occurred was not apparent from this study. A major advantage of the biochemical approach is the ability to measure substrate concentrations of adenosine triphosphate, creatine phosphate, and nicotinamide adenine dinucleotide, etc, which is virtually impossible histochemically.

Most of the ultrastructural work carried out so far has resulted in a purely descriptive assessment of myocardial damage, though Olsen (1973) and M. Sekiguchi (1975, personal communication) have used a scoring method to assess the degree of damage in HOCM and CoCM, respectively. We have started to use established morphometric techniques on electron microscope photographs in an attempt to quantify accurately changes in mitochondrial, sarcotubular, or myofibrillar proportions, or in any other cellular components. In this way, minor alterations in mitochondrial mass for example, which could pass unnoticed by simple inspection of photographs, may be detected and possibly correlated with other clinical or pathological features. It is possible that subtle effects of drugs may also be detected by this means. The use of the electron probe microanalysis (EMMA 4), as reported by Yarom et al. (1974), offers interesting possibilities for the investigation of intracellular 
ions of which calcium is the most obvious. Here also technical problems are considerable and the availability of this method is obviously limited so that progress may be slow.

We may draw several conclusions from this report of our experience of cardiac biopsy and the brief review of the analytical methods available. First, the method is safe and reliable but should be confined to a small number of investigators for maximum safety and productivity. Secondly, the diagnostic limitations are considerable but useful information can be valuable in selected cases. Thirdly, quantitative methods for electron microscopy, light microscopy, and histochemistry will make the information from each field more precise and thus of more comparative value. Finally, the application of biochemical analysis of biopsy tissue and other sophisticated techniques is more likely to produce the answer to the riddle of CoCM than simple morphological examination. At present we feel that the role of cardiac biopsy will remain extremely limited until better techniques for its examination are available; though we were initially enthusiastic, the clinical usefulness of the method has proved to be disappointingly restricted.

Professor Sleight is grateful to Drs. Sekiguchi and Konno for training in the use of their biopsy technique. We thank Dr. A. H. T. Robb-Smith for facilities and encouragement of this work, and our colleagues at the Radcliffe Infirmary for permission to report their cases.

\section{References}

Altmann, F. P. (1969). The quantitative elution of NitroBlue formazan from tissue sections. Histochemie, 17, 319326.

Bercu, B., Heinz, J., Choudhry, A. S., and Cabrera, P. (1964). Myocardial biopsy: a new technic utilizing the ventricular septum. American fournal of Cardiology, 14, 675-678.

Brooksby, I. A. B., Jenkins, B. S., Coltart, D. J., WebbPeploe, M. M., and Davies, M. J. (1974). Left-ventricular endomyocardial biopsy. Lancet, 2, 1222-1225.

Bulloch, R. T., Murphy, M. L., and Pearce, M. B. (1965). Intracardiac needle biopsy of the ventricular septum. American fournal of Cardiology, 16, 227-233.
Chayen, J., Bitensky, L., and Butcher, R. (1973). Practical Histochemistry. John Wiley, London.

Khan, M. A., Papadimitriou, J. M., Holt, P. G., and Kakulas, B. A. (1972). A calcium-citro-phosphate technique for the histochemical localization of myosin ATPase. Stain Technology, 47, 277-281.

Konno, S., Sekiguchi, M., and Sakakibara, S. (1971). Catheter biopsy of the heart. Radiologic Clinics of North America, 9, 491-510.

MacKay, E. H., Brown, R., and Pickering, D. (1976). Cardiac biopsy in skeletal myopathy. Fournal of Pathology, 120, 35-42.

Olsen, E. G. J. (1973). Pathology of hypertrophic obstructive cardiomyopathy. In Recent Advances in Studies on Cardiac Structure and Metabolism, Vol. 2, pp. 197-212. Ed. by E. Bajusz and G. Rona. M.T.P., Lancaster.

Olsen, E. G. J. (1974). Diagnostic value of the endomyocardial bioptome. Lancet, 1, 658-660.

Peters, T. J., Brooksby, I. A. B., Webb-Peploe, M. M., Wells, G., Jenkins, B. S., and Coltart, D. J. (1976). Enzymic analysis of cardiac biopsy material from patients with valvular heart disease. Lancet, 1, 269-270.

Richardson, P. J. (1974). King's endomyocardial bioptome. Lancet, 1, 660-661.

Sakakibara, S., and Konno, S. (1962). Endomyocardial biopsy. Fapanese Heart fournal, 3, 537-543.

Sekiguchi, M., and Konno, S. (1969). Histopathological differentiation employing endomyocardial biopsy in the clinical assessment of primary myocardial disease. Fapanese Heart fournal, 10, 30-46.

Shirey, E. K., Hawk, W. A., Mukerii, D., and Effler, D. B. (1972). Percutaneous myocardial biopsy of the left ventricle. Circulation, 46, 112-122.

Somers, K., Hutt, M. S. R., Patel, A. K., and D'Arbela, P. G. (1971). Endomyocardial biopsy in diagnosis of cardiomyopathies. British Heart fournal, 33, 822-832.

Sutton, D. C., and Sutton, G. C. (1960). Needle biopsy of the human ventricular myocardium: review of 54 consecutive cases. American Heart fournal, 60, 364-370.

Sutton, G. C., Driscoll, J. F., Gunnar, R. M., and Tobin, J. R. (1964). Exploratory mediastinotomy in primary myocardial disease. Progress in Cardiovascular Diseases, 7, 83-97.

Weinberg, M., Fell, E. H., and Lynfield, J. (1958). Diagnostic biopsy of the pericardium and myocardium. Archives of Surgery, 76, 825-829.

Yarom, R., Peters, P. D., and Hall, T. A. (1974). Effect of glutaraldehyde and urea embedding on intracellular ionic elements. X-ray microanalysis of skeletal muscle and myocardium. Fournal of Ultrastructure Research, 49, 405418.

Requests for reprints to Dr. E. H. MacKay, Harkness Laboratories, Radcliffe Infirmary, Oxford OX2 6HE. 